\title{
Modeling the polarization of high-energy radiation from accreting black holes
}

\section{A case study of XTE $\mathbf{J 1 1 1 8 + 4 8 0}$}

\author{
F. L. Vieyro ${ }^{1,2}$, G. E. Romero ${ }^{1,2}$, and S. Chaty ${ }^{3,4}$ \\ ${ }^{1}$ Instituto Argentino de Radioastronomía (IAR, CCT La Plata, CONICET), C.C.5, (1984) Villa Elisa, Buenos Aires, Argentina \\ e-mail: florenciavieyro@gmail.com \\ 2 Facultad de Ciencias Astronómicas y Geofísicas, Universidad Nacional de La Plata, Paseo del Bosque s/n, \\ 1900 La Plata, Argentina \\ 3 AIM, UMR 7158 CEA/DSM-CNRS-Université Paris Diderot, Irfu/Service d'Astrophysique, Centre de Saclay, \\ 91191 Gif-sur-Yvette Cedex, France \\ ${ }^{4}$ Institut Universitaire de France, 103 boulevard Saint-Michel, 75005 Paris, France
}

Received 22 May 2015 / Accepted 29 December 2015

\begin{abstract}
Context. The high-energy emission (400 keV-2 MeV) of Cygnus X-1, which is the best-studied Galactic black hole, was recently found to be strongly polarized. The origin of this radiation is still unknown.

Aims. We suggest that the emission is the result of non-thermal processes in the hot corona around the accreting compact object and study the polarization of high-energy radiation that is expected for black hole binaries.

Methods. Two contributions to the total magnetic field were taken into account in our study: a small-scale random component related to the corona, and an ordered magnetic field associated with the accretion disk. The degree of polarization of gamma-ray emission for this particular geometry was estimated together with the angle of the polarization vector.

Results. We obtain that the configuration of corona plus disk can account for the high degree of polarization of gamma-rays that are detected in galactic black holes and does not need to invoke a relativistic jet. We make specific predictions for sources in a low-hard state. In particular, the model is applied to the transient source XTEJ1118+480. We show that if a new outburst of XTE J1118+480 is observed, then its gamma-ray polarization should be measurable by future instruments, such as ASTRO-H or the proposed ASTROGAM.
\end{abstract}

Key words. X-rays: binaries - gamma rays: general - polarization - stars: individual: XTE J1118+480

\section{Introduction}

The hard X-ray and soft gamma-ray spectra of several black holes binaries (BHBs) present two components: a power-law component with an exponential cutoff, which is the result of Comptonization of soft photons from the disk by a hot corona, and a steeper non-thermal spectrum up to $\mathrm{MeV}$ energies (e.g., McConnell et al. 2000; Ling \& Wheaton 2003, 2005; Cadolle Bel et al. 2006). The detection of a high degree of polarization $(67 \pm 30 \%)$, in the non-thermal tail of the high-mass X-ray binary Cygnus X-1 confirmed that these components have different emission processes (Laurent et al. 2011; Jourdain et al. 2012; Romero et al. 2014).

Different scenarios have been proposed to explain the origin of the high-energy component (Poutanen \& Coppi 1998; Zdziarski et al. 2012, 2014b; Romero et al. 2010; Vieyro \& Romero 2012). Ultimately, its nature still remains unknown.

The hybrid thermal and non-thermal corona model of Poutanen \& Coppi (1998) attributes the high-energy tail of BHBs to Comptonization of the soft photons of the disk by a non-thermal population of electrons. This model has been successful in reproducing the soft gamma-ray spectrum of accreting black holes, but Compton scattering is not consistent with the high polarization fraction measured by INTEGRAL (Laurent et al. 2011).

Highly polarized radiation must be produced in the presence of an ordered magnetic field. A relativistic jet therefore is the first candidate for the origin of the gamma-ray emission. Zdziarski et al. (2012) proposed that the soft gamma-ray radiation is generated in a relativistic jet (see also Zdziarski et al. 2014a,b). They studied synchrotron and self-Compton emission that was the result of non-thermal electrons. To reproduce the $\mathrm{MeV}$ component, they required an electron spectrum that is difficult to explain with diffusive acceleration processes. For models with more realistic particle distributions, the synchrotron fluxes at $E \sim \mathrm{MeV}$ are lower than those observed in Cygnus X-1. Pepe et al. (2015) recently obtained good fits to the $\mathrm{MeV}$ emission for several sets of parameters. Nevertheless, current models for the polarization of the jet have problems to reproduce the polarization angle measured in Cygnus X-1.

Romero et al. (2010) and Vieyro \& Romero (2012) studied the transport of non-thermal particles in the corona of Cygnus X-1 and successfully reproduced the emission detected by COMPTEL and INTEGRAL at MeV energies (see also Vieyro et al. 2012). Furthermore, Romero et al. (2014) showed that the same radiation from the corona can develop the high 
degree of polarization detected by Laurent et al. (2011). In this context, the synchrotron emission of secondary leptons is responsible for the polarized radiation.

We here analyze the influence of a given configuration of the magnetic field on the polarization of synchrotron radiation, expanding our previous work on the topic (Romero et al. 2014). We then apply the model to XTEJ $1118+480$. This is a transient source that was discovered during an outburst in 2000 (Remillard et al. 2000), and it was later detected in a second outburst in 2005 (Zurita et al. 2005). Its location at high latitude over the Galactic plane - where the interstellar absorption is lowallowed several multi-wavelength studies of both events (Chaty et al. 2003; Maitra et al. 2009; Brocksopp et al. 2010). As a result, its spectrum was well characterized, and it resembled the low-hard state of BHBs on both occasions.

Our article has the following structure: in Sect. 2 we explain the basic hypothesis of the model. We study the non-thermal particle interactions in the corona and compute the final spectral energy distribution (SED). In Sect. 3, we describe a specific geometry of the magnetic field and estimate the polarization degree and the polarization vector angle. In Sect. 4 we consider XTE J1118+480. We present our results in Sect. 4.3, discuss them in Sect. 5, and finally conclude in Sect. 6.

\section{Basic model}

We consider a spherical corona with a radius $R_{\mathrm{c}}$, and an accretion disk that is truncated at $r_{\text {in }}$. The ratio $r_{\text {in }} / R_{\mathrm{c}}$ is taken as 0.9 , which is a standard value for the low-hard state (Poutanen 1998). The corona is assumed to be magnetically dominated and static, where particles can be removed by diffusion (Bisnovatyi-Kogan \& Blinnikov 1977).

The hard X-ray photon density energy distribution of the corona, $n_{\mathrm{ph}}$, is represented as a power law with an exponential cutoff $E_{\mathrm{c}}$,

$n_{\mathrm{ph}}\left(E_{\mathrm{ph}}\right) \propto E_{\mathrm{ph}}^{\alpha} \mathrm{e}^{-E_{\mathrm{ph}} / E_{\mathrm{c}}}$.

The normalization constant can be obtained from the bolometric luminosity according to

$\frac{L_{\mathrm{c}}}{4 \pi R_{\mathrm{c}}^{2} c}=\int_{0}^{\infty} E_{\mathrm{ph}} n_{\mathrm{ph}}\left(E_{\mathrm{ph}}\right) \mathrm{d} E_{\mathrm{ph}}$.

The accretion disk is modeled as a multi-temperature black body, as described in Frank et al. (2002) and Vila et al. (2012). At every radius, the disk is in local thermal equilibrium, and it radiates as a black body of temperature $T(r)$. The temperature profile for a standard disk is given by Shakura \& Sunyaev (1973):

$T(r)=\frac{T_{\max }}{0.488}\left(\frac{r}{r_{\text {in }}}\right)^{-3 / 4}\left(1-\sqrt{\frac{r_{\text {in }}}{r}}\right)^{1 / 4}$,

where $T_{\max }$ is the maximum temperature of the disk. The integrated disk spectrum can be obtained as

$F_{\gamma}\left(E_{\gamma}\right)=2 \pi \frac{\cos i}{d^{2}} \int_{r_{\text {in }}}^{r_{\text {out }}} B\left(E_{\gamma}, T(r)\right) r \mathrm{~d} r$

where $i$ is the inclination angle (we consider that the accretion disk is parallel to the orbital plane), and $B\left(E_{\gamma}, T(r)\right)$ is the Planck function, given by

$B\left(E_{\gamma}, T(r)\right)=\frac{2}{c^{2} h^{3}} \frac{E_{\gamma}^{3}}{\exp \left[E_{\gamma} / k T(r)\right]-1}$.
The low-hard state of BHBs is usually associated with the presence of relativistic jets. These jets are thought to be launched by a magnetic mechanism (e.g., Blandford \& Payne 1982). Since in several systems the jet kinetic power is of approximately the same luminosity as the corona, we estimate the value of the random magnetic field in the corona by assuming equipartition between the magnetic energy density and the bolometric photon density of the corona (Bednarek \& Giovannelli 2007),

$$
\frac{B_{\mathrm{c}}^{2}}{8 \pi}=\frac{L_{\mathrm{c}}}{4 \pi R_{\mathrm{c}}^{2} c} .
$$

For a more detailed description of the corona model we refer to Romero et al. (2010) and Vieyro \& Romero (2012).

\subsection{Non-thermal particle injection}

A likely mechanism of particle acceleration in accreting black hole coronae is magnetic reconnection (e.g., de Gouveia Dal Pino et al. 2010; Lazarian et al. 2011). In this process particles undergo a Fermi-type acceleration in magnetic reconnection sites, which are regions where the topology of the magnetic field changes because magnetic fluxes of opposed polarity approach each other. We consider an efficiency for this mechanism of $\eta \sim 0.01$ (Vieyro \& Romero 2012). The resulting particle spectrum for a diffusive acceleration mechanism is a power law. Accordingly, we adopt an injection function of primary particles given by

$Q(E) \propto E^{-\Gamma} \mathrm{e}^{-E / E_{\max }}$,

where $E_{\max }$ is the maximum energy, and it is determined by a balance between the cooling rates and the acceleration rate. The normalization constant depends on the available energy to accelerate particles, $L_{\mathrm{rel}}$. We adopt $q=L_{\mathrm{rel}} / L_{\mathrm{c}}=0.1$, a value within a reasonable range in the parameter space (del Valle et al. 2011; Vieyro \& Romero 2012). It is unknown how the energy is distributed between hadrons and leptons. It is useful to define the parameter $a$ as the ratio of power injected in protons and electrons, that is, $a=L_{\mathrm{p}} / L_{\mathrm{e}}$. We adopt $a=100$, as observed in galactic cosmic rays (Ginzburg \& Syrovatskii 1964). The value of $a$, however, does not affect the final particle distribution index because it has no effect on the estimates of the polarization degree.

\subsection{Particle transport}

When particles are injected, they interact with the different local fields in the source (i.e., photon, matter, and magnetic fields). We study the transport of primary and secondary (pions, muons, and electron-positron pairs) particles and the transport of photons, using the numerical method described in Vieyro \& Romero (2012).

Figure 1 shows the final SED, computed for three values of the injection index $\Gamma$ : the standard value $\Gamma=2$, and $\Gamma=1.5,2.5$ which are intermediate values that can be obtained with a mechanism based on magnetic reconnection acceleration (Drury 2012; Bosch-Ramon 2012).

The gap that appears at $\sim 10^{8} \mathrm{eV}<E_{\gamma}<10^{13} \mathrm{eV}$ is the result of absorption in the internal field of the corona. Absorption by photon annihilation has a fundamental role in our model; on the one hand, it shapes the final SED at MeV energies, and on the other hand, it is the main source of secondary pairs in the corona.

The polarized radiation measured by INTEGRAL was at $400 \mathrm{keV}<E_{\gamma}<2 \mathrm{MeV}$; the main contribution to the final flux in this energy range is the synchrotron emission of secondary pairs. 
Table 1. Main parameters of the corona and the disk.

\begin{tabular}{lc}
\hline \hline Parameters & Value \\
\hline$L_{\mathrm{c}}:$ corona luminosity $\left[\mathrm{erg} \mathrm{s}^{-1}\right]$ & $10^{36}$ \\
$k T_{\max }:$ maximum disk temperature $[\mathrm{eV}]$ & 22.0 \\
$r_{\mathrm{in}}:$ inner disk radius $\left[r_{\mathrm{g}}\right]$ & 160.0 \\
$r_{\mathrm{in}} / R_{\mathrm{c}}:$ inner disk/corona ratio & 0.9 \\
$\alpha: \mathrm{X}$-ray spectrum power-law index & 1.75 \\
$E_{\mathrm{c}}:$ X-ray spectrum cutoff $[\mathrm{keV}]$ & 200 \\
$B_{\mathrm{c}}:$ magnetic field $[\mathrm{G}]$ & $10^{4}$ \\
$n_{i}$, ne: plasma density $\left[\mathrm{cm}^{-3}\right]$ & $10^{11}$ \\
\hline
\end{tabular}

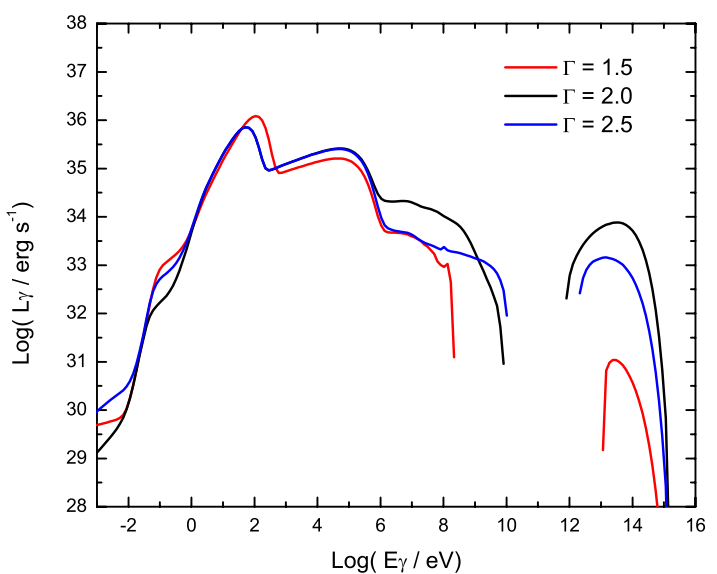

Fig. 1. Spectral energy distributions for different values of the primary injection index.

\section{Polarization}

An isotropic distribution of relativistic leptons with a power-law energy spectrum $N(E) \propto E^{-\Gamma}$ in a region with a large-scale magnetic field produces a synchrotron emission that is linearly polarized (Korchakov \& Syrovatskii 1962). In the presence of a uniform magnetic field $B_{\mathrm{u}}$, the degree of linear polarization is (e.g., Pacholczyk \& Swihart 1970)

$P_{0}(\Gamma)=\frac{\Gamma+1}{\Gamma+7 / 3}$

As an example, Fig. 2 shows the secondary pair distributions corresponding to the spectra of Fig. 1. For a primary injection index of $\Gamma=1.5,2,2.5$, the secondary pair index is $\Gamma_{\mathrm{e}^{ \pm}}=2.7,3.0$, and 3.6 , respectively. If the magnetic field were completely uniform, the polarization degree would be $73-77 \%$.

This is an upper limit to the degree of polarization, however, since the non-uniformity of the magnetic field tends to decrease the value. For a magnetic field with a random component $B_{\mathrm{c}}$ and a spatial scale of variation smaller than the source size, the degree of polarization is reduced to (Burn 1966):

$P(\Gamma)=P_{0}(\Gamma) \frac{B_{\mathrm{u}}^{2}}{B_{\mathrm{u}}^{2}+B_{\mathrm{c}}^{2}}$.

In BHBs, the random magnetic field is associated with the corona, whereas the uniform component is related to the accretion disk. Since the disk only penetrates the corona up to a certain radius, we consider that $B_{\mathrm{u}}=\xi B_{\mathrm{d}}$, where $B_{\mathrm{d}}$ is the magnetic field of the disk, and $\xi$ is a filling factor that depends on the fraction of the corona volume covered by $B_{\mathrm{d}}$. In zeroth-order approximation, $\xi=1-\left(r_{\text {in }} / R_{\mathrm{c}}\right)^{3}$.

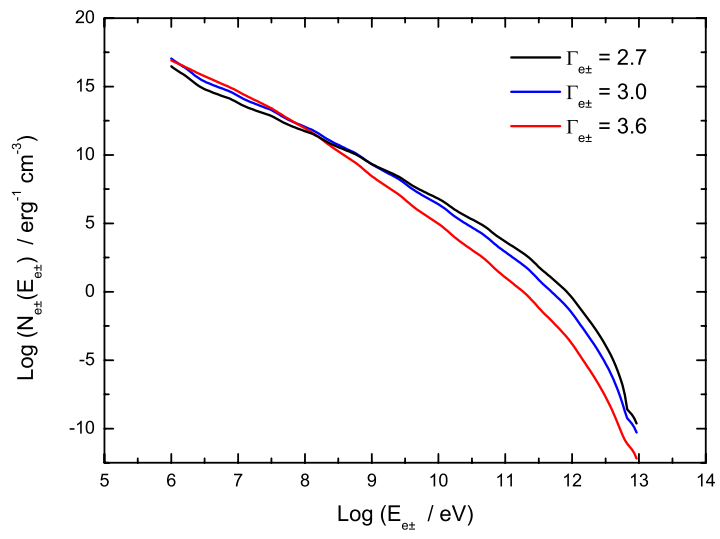

Fig. 2. Secondary pair distributions for the different values of the primary injection index.

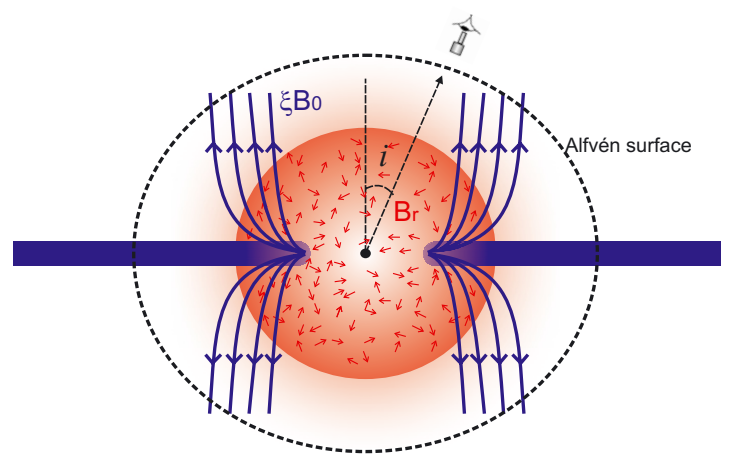

Fig. 3. Illustration of the geometry of the magnetic field components.

We also consider that the angular distribution of the magnetic field, $B_{\mathrm{u}}(\Omega)$, has axial symmetry (see Fig. 3 for a schematic representation). If $B_{\|}$and $B_{\perp}$ are the components of the magnetic field of the disk parallel and normal to the symmetry axis, the observed degree of polarization can be obtained as (Korchakov \& Syrovatskii 1962)

$P_{\text {tot }}(\Gamma)=P_{0} \frac{15}{8} \frac{\Gamma+5}{\Gamma+7} \frac{B_{\mathrm{u}}^{2}}{B_{\mathrm{u}}^{2}+B_{\mathrm{c}}^{2}} \frac{\left|\left\langle\Delta B^{2}\right\rangle\right|}{B_{\mathrm{u}}^{2}} \sin ^{2} i$,

where $i$ is the angle between the symmetry axis and the line of sight $^{1},\left\langle\Delta B^{2}\right\rangle=\left\langle B_{\perp}^{2}\right\rangle-\left\langle B_{\|}^{2}\right\rangle$, and the averaging is carried over out as

$\langle B\rangle=\frac{1}{4 \pi} \int B_{\mathrm{u}}(\Omega) \mathrm{d} \Omega$.

For the magnetic field of the accretion disk, we use the geometry described in Stepanovs \& Fendt (2014). The potential vector is defined as $\boldsymbol{A}=A \breve{e}_{\phi}$, where

$A=\frac{4}{3} \frac{B_{0}}{r^{1 / 4}} \frac{1}{\left[1+4 \cot ^{2} \theta\right]^{5 / 8}}$

1 In a previous work by Romero et al. (2014), the factor $\sin ^{2} i$ was not included in Eq. (10). This equation was used to constrain the ratio $B_{\mathrm{u}} / B_{\mathrm{r}}$ in the low-hard state. Although this ratio is affected by the missing factor, the main result of expecting a higher polarization degree in an intermediate state than in the low-hard state remains valid. 


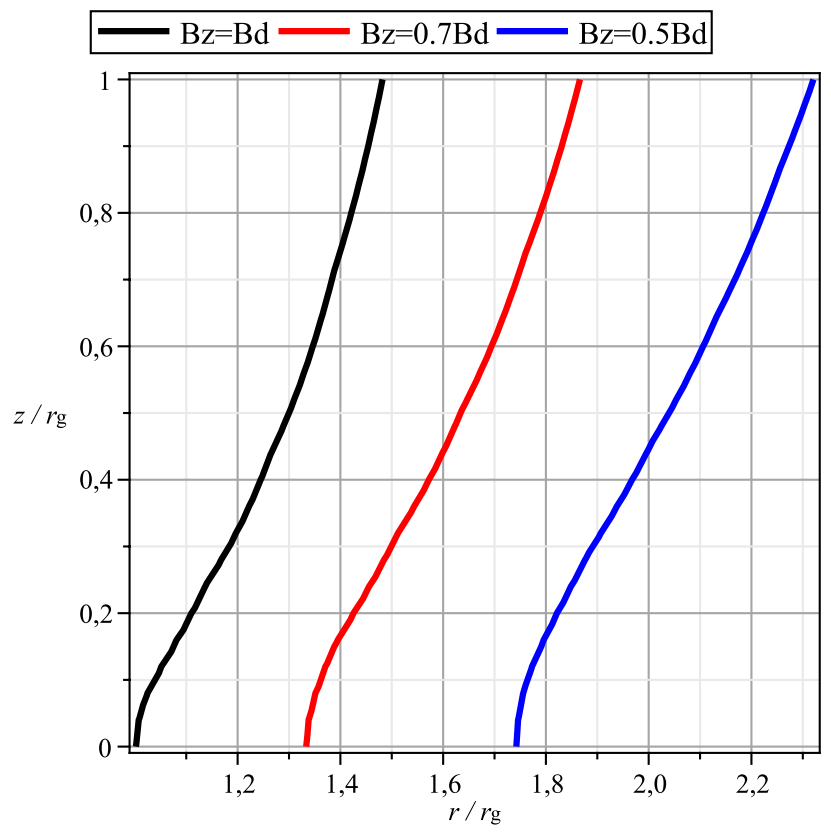

Fig. 4. Parallel component of the magnetic field associated with the accretion disk. Both axes are normalized to $r_{\text {in }}$, and $B_{\mathrm{d}}=B_{\mathrm{d}}\left(r=r_{\text {in }}, \theta=\right.$ $\pi / 2)$.

The magnetic field is $\boldsymbol{B}=\boldsymbol{\nabla} \times \boldsymbol{A}$, and its components are

$$
\begin{aligned}
& B_{r}=\frac{4 B_{0}}{3 r^{5 / 4}} \frac{\cot \theta}{\left(1+4 \cot ^{2} \theta\right)^{5 / 8}}\left[1+\frac{5\left(1+\cot ^{2} \theta\right)}{\left(1+4 \cot ^{2} \theta\right)}\right], \\
& B_{\theta}=-\frac{B_{0}}{r^{5 / 4}}\left(1+4 \cot ^{2} \theta\right)^{-5 / 8} \\
& B_{\phi}=0
\end{aligned}
$$

where $B_{0}$ is obtained through the condition $B\left(r_{\mathrm{in}}, \pi / 2\right)=B_{\mathrm{d}}$. The geometry represented by Eq. (13) fulfills the necessary conditions for the magnetic field to launch an outflow. Inside the Alfvén region, the force-free approximation holds, and the field lines corotate with the disk. Only outside the Alfvén radius is the azimuthal component relevant, since the force-free approximation is no longer valid. We therefore adopt an azimuthal component of the magnetic field equal to zero in the inner region (e.g., Spruit 2010).

To estimate the parallel and perpendicular components of the magnetic field, we first transform the spherical components given by Eq. (13) to Cartesian components, and then to cylindrical ones. Figure 4 shows the contour lines of the resulting parallel component of the field.

The launching of an outflow requires a minimum inclination angle of the field lines with respect to the symmetry axis of $\theta=$ $30^{\circ}$ (Blandford \& Payne 1982). Therefore the quantity $\left\langle\Delta B^{2}\right\rangle$ is estimated using Eq. (11), with $30^{\circ}<\theta<90^{\circ}$, and results in $\left\langle\Delta B^{2}\right\rangle / B_{\mathrm{u}}^{2} \sim 0.23$.

The factor $\left\langle\Delta B^{2}\right\rangle / B_{\mathrm{u}}^{2}$ decreases when the inclination of the $B_{z}$ component increases. Then, for a magnetic field that is dragged by the accretion disk to the compact object, the inclination of the field lines becomes larger, resulting in an increment of the polarization degree. This effect could be important in the high-soft state.

On the other hand, the polarization degree strongly depends on the inclination angle of the binary system: for an edge-on system (i.e., $i=0$ ) we expect the maximum possible degree of polarization, whereas for a face-on disk the degree of polarization should be negligible.

\section{Application to XTE J1118+480}

\subsection{Multi-wavelength observations of XTE J1118+480 in outbursts}

The soft X-ray transient XTEJ1118+480 was discovered by RXTE on 2000 March 29 at the Galactic coordinates $(l, b)=$ $(157.62 \mathrm{deg},+62.32 \mathrm{deg})$ as a weak $(39 \mathrm{mCrab})$ slowly rising X-ray source (Remillard et al. 2000). The post-analysis revealed an outburst in January 2000, with a similar brightness.

The location of XTEJ1118+480 at an unusually high Galactic latitude in the direction of the Lockman Hole implied a very low absorption along the line of sight $\left(N_{\mathrm{H}} \sim 0.80-1.30 \times\right.$ $10^{20} \mathrm{~cm}^{-2}$, Chaty et al. 2003). This low interstellar absorption allowed harvesting an unprecedented wealth of multi-wavelength coverage from radio to X-ray domains, including even the first extreme-ultraviolet spectrum of an X-ray transient (see Chaty et al. 2003, and references therein).

Optical observations of the source in quiescence led to the determination of a large value of the mass function, $f(M)=$ $5.9 \pm 0.4 M_{\odot}$, making this source a strong black-hole candidate (Wagner et al. 2001; McClintock et al. 2001). The companion star is a low-mass star of $M=0.27 \pm 0.05 M_{\odot}$ and has a spectral type between K5V and K8V (Chaty et al. 2003; Gallo et al. 2014). XTE J1118+480 exhibits an orbital period of $4.1 \mathrm{~h}=$ $0.17082 \mathrm{~d}$, one of the shortest of all black-hole candidates (Cook et al. 2000).

The distance to the source is estimated as $d=1.72 \mathrm{kpc}$, and the inclination angle of the disk with respect to the line of sight is $\sim 70^{\circ}$ (Gelino et al. 2006).

This black-hole X-ray transient displayed two outbursts: the first in 2000, the second in 2005. Thanks to the low interstellar absorption, it was observed each time from radio to hard X-rays. In the low-hard X-ray spectral state the source exhibited correlated X-ray and radio behavior during both outbursts. The light curves, however, showed different behaviors as follows.

i) During the 2000 outburst the source stayed in a very low low-hard state, with an inner radius of the accretion disk estimated at $\sim 350 R_{\mathrm{S}}\left(R_{\mathrm{S}}=\right.$ Schwarzschild radius for an object of mass $M$ ), and a strong non-thermal (synchrotron) contribution in the optical and near-infrared (Chaty et al. 2003). The SED of the source (from radio to X-rays) remained barely unchanged for almost three months. This long plateau-like phase was probably due to a stable jet.

ii) The 2005 outburst was more typical of a canonical soft X-ray transient, with a more substantial contribution from the accretion disk, and a fast rise, exponential decay (FRED) light-curve (see Zurita et al. 2005; Brocksopp et al. 2010 and references therein).

The different characteristics of these two outbursts make XTE J1118+480 a very promising source on which to study polarization and to determine its origin.

\subsection{Corona of XTE J1118+480}

We characterize the hard X-ray emission of the corona during the 2000 outburst as a power law of index $\alpha \sim 1.75$ and an 
Table 2. Main parameters of the corona of XTE J1118+480 during its outbursts.

\begin{tabular}{lcc}
\hline \hline Parameters & Outburst & Outburst \\
& 2000 & 2005 \\
\hline$L_{\mathrm{c}}:$ corona luminosity $\left[\mathrm{erg} \mathrm{s}^{-1}\right]$ & $1.3 \times 10^{36}$ & $9.0 \times 10^{35}$ \\
$k T_{\text {max }}:$ maximum disk temperature $[\mathrm{keV}]$ & 22.0 & 45.0 \\
$r_{\text {in }}:$ inner disk radius $\left[r_{\mathrm{g}}\right]$ & 160.0 & 50.0 \\
$r_{\text {in }} / R_{\mathrm{c}}:$ inner disk/corona ratio & 0.9 & 0.9 \\
$\alpha:$ X-ray spectrum power-law index & 1.75 & 1.78 \\
$E_{\mathrm{c}}:$ X-ray spectrum cut-off $[\mathrm{keV}]$ & 200 & 200 \\
$B_{\mathrm{c}}:$ magnetic field $[\mathrm{G}]$ & $2.6 \times 10^{4}$ & $1.1 \times 10^{5}$ \\
$n_{i}, n_{e}:$ plasma density $\left[\mathrm{cm}^{-3}\right]$ & $1.3 \times 10^{11}$ & $2.4 \times 10^{12}$ \\
\hline
\end{tabular}

exponential cutoff at $E_{\mathrm{c}}=200 \mathrm{keV}$ (McClintock et al. 2001; Chaty et al. 2003). The luminosity at $1<E<160 \mathrm{keV}$ was $L_{\mathrm{c}}=1.31 \times 10^{36} \mathrm{erg} \mathrm{s}^{-1}$ (McClintock et al. 2001).

According to Maitra et al. (2009), the 2005 outburst was fainter, with a steeper power-law in the X-rays. For this event, we consider a coronal luminosity of $L_{\mathrm{c}}=9.0 \times 10^{35} \mathrm{erg} \mathrm{s}^{-1}$ and a power-law of index $\alpha \sim 1.78$.

The values of $r_{\text {in }}$, and $k T_{\max }$ determine the spectrum of the accretion disk, and they were varied to obtain the best-fit model in each case. The parameters $L_{\mathrm{c}}, \alpha$, and $E_{\mathrm{c}}$ are inferred from observations. The remaining parameters $\left(B_{\mathrm{c}}, n_{e, i}\right)$ are determined by applying the model discussed in Sect. 2 , and their values are presented in Table 2 for both outbursts.

In a corona characterized by these parameters, we inject populations of non-thermal particles, both electrons and protons. We adopt the standard index of $\Gamma=2$ for the particle injection function (see the previous section for a discussion on the incidence of the particle index on the polarization degree). There were no observations of the source during the outbursts at $\mathrm{MeV}$ energies. According to our model, this is the energy range where the synchrotron radiation of secondary pairs dominates the spectrum. Since the content of pairs is related to the hadronic content in the source, the parameters $\eta, q$, and $a$ cannot be completely determined without simultaneous observations at $\mathrm{TeV}$ energies. Nevertheless, they are constrained by the magnetic energy density available for accelerating particles by magnetic reconnection. We adopt $\eta=0.01$ and $q=0.1$; these are values within the allowed range of space parameters (e.g., Vieyro \& Romero 2012). For the proton-to-lepton ratio we use $a=100$, as observed in galactic cosmic rays. Changes in these last three variables do not affect the predictions made on the polarization degree, however. Observations in the $\mathrm{MeV}-\mathrm{TeV}$ energy range might be used to set stronger constraints on these parameters during future outbursts.

\subsection{Results}

Figure 5 shows the SEDs for the two outburst of XTEJ1118+480, together with the data from different instruments. The corresponding final secondary pair distributions are shown in Fig. 6. In both cases, the index of the pair distributions is $\sim 3$. We note that the radio emission in our model is produced in the jet and hence is not calculated here.

The magnetic field at the base of the jet, $B_{\text {jet }}\left(z_{0}\right)$, was estimated by Vila et al. (2012) for both outbursts. The estimated values are $B_{\text {jet }}\left(z_{0}\right)=1.3 \times 10^{7} \mathrm{G}, 7.9 \times 10^{6} \mathrm{G}$, with $z_{0}=50 r_{\mathrm{g}}$, for the 2000 and 2005 outbursts, respectively. To obtain a value for the magnetic field in the disk inner radius, we extrapolate the
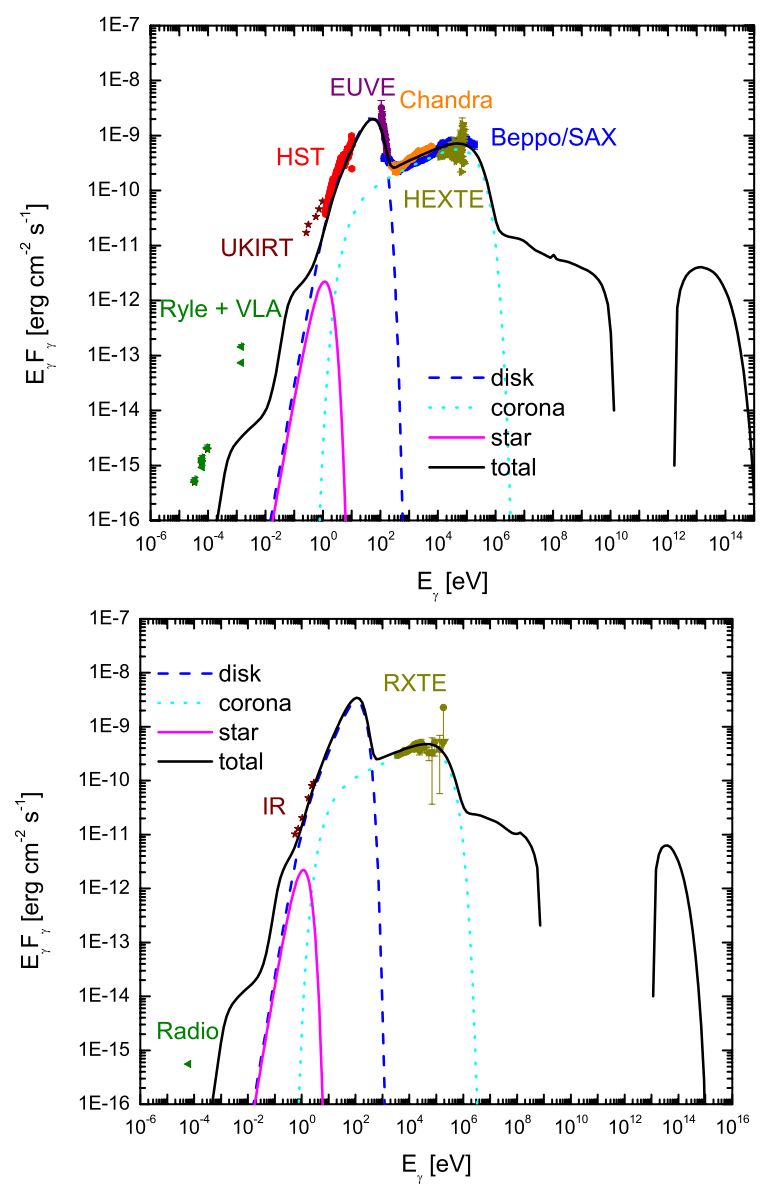

Fig. 5. Top panel: computed SED for the 2000 outburst. Data from Chaty et al. (2003). Bottom panel: SED of the 2005 outburst. Data from Brocksopp et al. (2010).

results of Vila et al. (2012) using the following expression:

$B_{\mathrm{d}}=B_{\text {jet }}\left(z_{0}\right)\left(\frac{z_{0}}{r_{\mathrm{in}}}\right)^{5 / 4}$

where the dependence $B_{\theta} \propto\left(R_{\mathrm{in}} / r\right)^{5 / 4}$ is only valid for the nonnull component of the magnetic field on the plane of the disk (i.e., $\theta=\pi / 2$ ).

Finally, we compute the degree of polarization with the model presented in Sect. 3 and obtain $\sim 23 \%$ for both outbursts.

The degree of polarization obtained from Eq. (10) depends mainly on the intrinsic properties of the synchrotron radiation. The geometry of the magnetic field only affects it through the factor $\left\langle\Delta B^{2}\right\rangle=\left\langle B_{\perp}^{2}\right\rangle-\left\langle B_{\|}^{2}\right\rangle$, as we discussed in Sect. 3 .

The angle of the polarization vector $\chi_{E}$ is strongly affected by the configuration of the magnetic field. To obtain the Stokes parameters $Q$ and $U$, we follow the method presented in Nalewajko \& Sikora (2012).

We adopt a Cartesian coordinate system $(x, y, z)$, in which the accretion disk axis (and consequently the magnetic field symmetry axis) is oriented along the $z$-axis. In this system, the unity vector $\breve{\boldsymbol{k}}=(\sin i, 0, \cos i)$ determines the direction to the observer. An orthogonal system can be defined in the image plane by the unity vectors

$\breve{\boldsymbol{v}}=(\cos i, 0,-\sin i)$,

$\breve{w}=(0,1,0)$. 

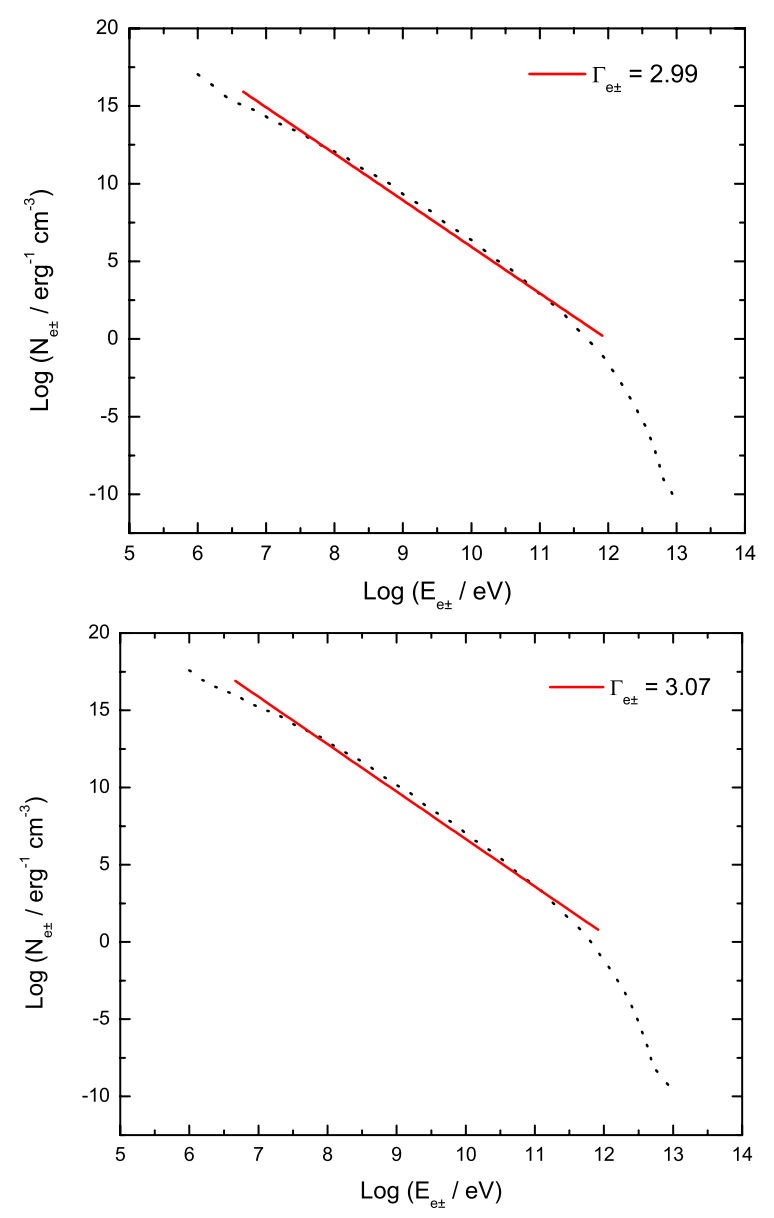

Fig. 6. Secondary pair distribution for the 2000 outburst (top panel) and the 2005 outburst (bottom panel). The solid line indicates the best power-law fit.

The Stokes parameters are then obtained as

$$
\begin{aligned}
& Q=I P_{\text {tot }} \cos \left(2 \chi_{E}\right)=\frac{C P_{\text {tot }}}{4 \pi} \int \mathrm{d} \Omega\left[(\boldsymbol{B} \cdot \breve{\boldsymbol{w}})^{2}-(\boldsymbol{B} \cdot \breve{\boldsymbol{v}})^{2}\right], \\
& U=I P_{\text {tot }} \sin \left(2 \chi_{E}\right)=\frac{C P_{\text {tot }}}{4 \pi} \int \mathrm{d} \Omega[-2(\boldsymbol{B} \cdot \breve{\boldsymbol{w}})(\boldsymbol{B} \cdot \breve{\boldsymbol{v}})],
\end{aligned}
$$

where $C$ is a constant that depends on the intensity $I$ of synchrotron radiation. Using the Cartesian components of the magnetic field resulting from Eq. (13), we obtain $U=0$ and $Q<0$; this implies $\chi_{E}=90^{\circ}$, as expected, since the angle is measured from the projected direction of the magnetic field symmetry axis.

\section{Discussion}

The degree of polarization that we obtained here for XTE J1118+480 ( 21\%) is significantly lower than the one measured in Cygnus X-1 (67-76\%; Laurent et al. 2011; Jourdain et al. 2012). The fact that the accretion rate onto the black hole of Cygnus X-1 is very stable over years (the observations by INTEGRAL covered a period of $6.5 \mathrm{yr}$ ) might be the reason for the high degree of polarization in this source (Russell \& Shahbaz 2014). We might expect therefore that a transient source such as XTE J1118+480, where the magnetic field cannot reach such an ordered configuration, presents a lower polarization degree than Cygnus X-1.

The mechanism responsible of the presence of non-thermal particles in coronae is thought to be magnetic reconnection.
Fast magnetic reconnection events have been studied for several structures of the disks, resulting in the acceleration of particles up to relativistic energies (e.g., de Gouveia Dal Pino et al. 2010; Singh et al. 2015; Khiali et al. 2015). The basic idea is that a first-order Fermi mechanism takes place within the reconnection zone caused by two converging magnetic fluxes of opposite polarity (de Gouveia dal Pino \& Lazarian 2005). The resulting injection function of relativistic particles is a power law with an index in the range of $1 \leq \Gamma \leq 3$ (Drury 2012). The value of the spectral index is related to the compression of the plasma, where a higher compression leads to a harder injection function. Given the dependence of the polarization degree of synchrotron radiation on the particle index (Eq. (8)), a softer particle distribution should result in a higher degree of polarization.

Changes in the accretion rate are related to the spectral state of the source. The low-hard state takes place for low values of $\dot{m}$; for higher values, the source switches to a high state, in which our model predicts a higher degree of polarization. This is mainly because of two factors: on the one hand, the disk extends up to the last stable orbit in the high-soft state, so the ordered magnetic field covers a higher volume fraction of the corona (i.e., $\xi$ increases). On the other hand, we obtain that the degree of polarization decreases for a high inclination of the lines of the magnetic field of the disk. Then, the polarization degree should be lower in the low-hard than in the high-soft state. This is the opposite result of what would be expected if the polarized gamma radiation had its origin in a relativistic jet, since jets are not produced in the high-soft state. In addition, the angle of polarization is also expected to change in the high-soft state. The magnetic field lines are inclined in the coronal region, so the polarization should also change direction from perpendicular (as obtained in Sect. 4.3, $\left.\chi_{E}=90^{\circ}\right)$ to parallel $\left(\chi_{E}=0^{\circ}\right)$.

There is no dedicated gamma-ray polarimeter currently in space, but different instruments that could test the predictions of our model are planned for the near future. This is the case of the Soft Gamma-ray Detector (SGD) onboard ASTRO-H, an instrument that will be sensitive to $<10 \%$ polarization in the 50-200 keV energy band, and it is planned to be launched in 2015 (Tajima et al. 2010). The missions of ESA, DUAL and GRI (Gamma-Ray Imager), also include high-energy polarimetric observations, and the French National Research Agency (CNRS) proposes to build a gamma-ray detector and polarimeter in the $\mathrm{MeV}-\mathrm{GeV}$ energy range (project HARPO, standing for Hermetic Argon Polarimeter). In addition, the ASTROGAM space project is designed to study both steady and transient sources in the $0.3 \mathrm{MeV}-1 \mathrm{GeV}$ energy range; this will improve the sensitivity by a factor $10-30$ in the range $0.3-30 \mathrm{MeV}$ with respect to previous missions. It is also planned to surpass Fermi's sensitivity. This mission will be equipped with polarimetric facilities at $E>\mathrm{MeV}$ (Tavani 2015).

\section{Conclusions}

We have studied the polarization of synchrotron radiation of electron-positron pairs in the corona of a BHB in the low-hard state. In this state, the magnetic field of the disk is able to launch an outflow. We adopted a specific angular distribution for this ordered field, and we also estimated the random magnetic field of the corona. We applied our model to the source XTE J1118+480, whose unique set of data allowed us to accurately test our models and constrain polarization predictions. Taking the two contributions to the total magnetic field into account, we obtained a degree of polarization of $\sim 21 \%$ for a state with similar characteristics to those observed in the 2000 and 2005 outbursts of 
XTEJ1118+480. According to our model, if the source were again observed in outburst, it should present polarization in the energy range of $0.1-10 \mathrm{MeV}$, which will be detectable by future instruments.

At the time XTEJ1118+480 was observed, no dedicated instruments operated at $\mathrm{MeV}$ energies. Our model predicts polarized $\mathrm{MeV}$ emission and simultaneous unpolarized $\mathrm{TeV}$ radiation for this source. Since this Galactic source is located in a privileged low-absorption region and undergoes relatively frequent outbursts, our quantitative predictions might be a valuable tool to test a coronal non-thermal model through future multiwavelength observations.

The presence of polarized gamma-ray radiation in the high state of XRBs would strongly support our model over those proposing the origin of the $\mathrm{MeV}$ tail emission in the jet.

Acknowledgements. F.L.V. acknowledges Carolina Pepe and Ileana Andruchow for helpful discussions. This work was supported by the Argentine Agency ANPCyT (PICT 00878), as well as by grant AYA2013-47447-C3-1-P (Spain). S.C. and F.L.V. acknowledge funding by the Sorbonne Paris Cite (SPC), Scientific Research Project - Argentina 2014. S.C. acknowledges funding from the Centre National d'Études Spatiales (CNES). This work is based on observations obtained with MINE (the Multi-wavelength INTEGRAL NEtwork), supported by the CNES.

\section{References}

Bednarek, W., \& Giovannelli, F. 2007, A\&A, 464, 437

Bisnovatyi-Kogan, G. S., \& Blinnikov, S. I. 1977, A\&A, 59, 111

Blandford, R. D., \& Payne, D. G. 1982, MNRAS, 199, 883

Bosch-Ramon, V. 2012, A\&A, 542, A125

Brocksopp, C., Jonker, P. G., Maitra, D., et al. 2010, MNRAS, 404, 908

Burn, B. J. 1966, MNRAS, 133, 67

Cadolle Bel, M., Sizun, P., Goldwurm, A., et al. 2006, A\&A, 446, 591

Chaty, S., Haswell, C. A., Malzac, J., et al. 2003, MNRAS, 346, 689

Cook, L., Patterson, J., Buczynski, D., \& Fried, R. 2000, IAU Circ., 7397, 2

de Gouveia dal Pino, E. M., \& Lazarian, A. 2005, A\&A, 441, 845

de Gouveia Dal Pino, E. M., Piovezan, P. P., \& Kadowaki, L. H. S. 2010, A\&A, 518, A5

del Valle, M. V., Romero, G. E., Luque-Escamilla, P. L., Martí, J., \& Ramón

Sánchez-Sutil, J. 2011, ApJ, 738, 115

Drury, L. O. 2012, MNRAS, 422, 2474
Frank, J., King, A., \& Raine, D. J. 2002, Accretion Power in Astrophysics: Third Edition (Cambridge, UK: Cambridge University Press)

Gallo, E., Miller-Jones, J. C. A., Russell, D. M., et al. 2014, MNRAS, 445, 290

Gelino, D. M., Balman, Ş., Kızıloğlu, Ü., et al. 2006, ApJ, 642, 438

Ginzburg, V. L., \& Syrovatskii, S. I. 1964, The Origin of Cosmic Rays, ed. D. Ter Harr (New York: Macmillan, A Pergamon Press Book), Translated by H. S. H. Massey

Jourdain, E., Roques, J. P., \& Malzac, J. 2012, ApJ, 744, 64

Khiali, B., de Gouveia Dal Pino, E. M., \& del Valle, M. V. 2015, MNRAS, 449,34

Korchakov, A. A., \& Syrovatskii, S. I. 1962, Sov. Ast., 5, 678

Laurent, P., Rodriguez, J., Wilms, J., et al. 2011, Science, 332, 438

Lazarian, A., Kowal, G., Vishniac, E., \& de Gouveia Dal Pino, E. 2011, Planet. Space Sci., 59, 537

Ling, J. C., \& Wheaton, W. A. 2003, ApJ, 584, 399

Ling, J. C., \& Wheaton, W. A. 2005, ApJ, 622, 492

Maitra, D., Markoff, S., Brocksopp, C., et al. 2009, MNRAS, 398, 1638

McClintock, J. E., Haswell, C. A., Garcia, M. R., et al. 2001, ApJ, 555, 477

McConnell, M. L., Ryan, J. M., Collmar, W., et al. 2000, ApJ, 543, 928

Nalewajko, K., \& Sikora, M. 2012, A\&A, 543, A115

Pacholczyk, A. G., \& Swihart, T. L. 1970, ApJ, 161, 415

Pepe, C., Vila, G. S., \& Romero, G. E. 2015, A\&A, 584, A95

Poutanen, J. 1998, in Theory of Black Hole Accretion Disks, eds. M. A. Abramowicz, G. Bjornsson, \& J. E. Pringle, Cambridge Contemporary Astrophysics (Cambridge, UK: Cambridge University Press), 100

Poutanen, J., \& Coppi, P. S. 1998, Phys. Scr. T, 77, 57

Remillard, R., Morgan, E., Smith, D., \& Smith, E. 2000, IAU Circ., 7389, 2

Romero, G. E., Vieyro, F. L., \& Vila, G. S. 2010, A\&A, 519, A109

Romero, G. E., Vieyro, F. L., \& Chaty, S. 2014, A\&A, 562, L7

Russell, D. M., \& Shahbaz, T. 2014, MNRAS, 438, 2083

Shakura, N. I., \& Sunyaev, R. A. 1973, A\&A, 24, 337

Singh, C. B., de Gouveia Dal Pino, E. M., \& Kadowaki, L. H. S. 2015, ApJ, 799, L20

Spruit, H. C. 2010, in Lect. Notes Phys., ed. T. Belloni (Berlin: Springer Verlag), 794, 233

Stepanovs, D., \& Fendt, C. 2014, ApJ, 793, 31

Tajima, H., Blandford, R., Enoto, T., et al. 2010, in SPIE Conf. Ser., 7732

Tavani, M. 2015, The gamma-ray sky with Astrogam: Second astrogam worshop

Vieyro, F. L., \& Romero, G. E. 2012, A\&A, 542, A7

Vieyro, F. L., Sestayo, Y., Romero, G. E., \& Paredes, J. M. 2012, A\&A, 546, A46

Vila, G. S., Romero, G. E., \& Casco, N. A. 2012, A\&A, 538, A97

Wagner, R. M., Foltz, C. B., Shahbaz, T., et al. 2001, ApJ, 556, 42

Zdziarski, A. A., Lubiński, P., \& Sikora, M. 2012, MNRAS, 423, 663

Zdziarski, A. A., Pjanka, P., Sikora, M., \& Stawarz, Ł. 2014a, MNRAS, 442, 3243

Zdziarski, A. A., Stawarz, Ł., Pjanka, P., \& Sikora, M. 2014b, MNRAS, 440, 2238

Zurita, C., Rodríguez, D., Rodríguez-Gil, P., et al. 2005, ATel, 383, 1 